\title{
Electrical Conduction of Polypeptides as Related to Molecular Structure
}

\author{
Sumio HiRoTA \\ Materials Division, Electrotechnical Laboratory, \\ 1-1-4 Umezono, Sakura-mura, Niihari-gun, \\ Ibaraki 305, Japan
}

(Received October 31, 1981)

\begin{abstract}
The temperature dependence of electric conductivity, the frequency and temperature dependence of the dielectric constant, and the hydrogen-deuterium exchange reaction of the amide group were studied for solid poly $(\gamma$-methyl L-glutamate), poly( $\gamma$-benzyl L-glutamate), and $\operatorname{poly}(\beta$-benzyl L-aspartate). A linear relation was found between the activation energy for conductivity and the reciprocal of the static dielectric constant, and was interpreted in terms of the carrier generation process. The conductivity at the glass-like transition temperature was about $3 \times 10^{-18} \mathrm{ohm}^{-1} \mathrm{~cm}^{-1}$. The generation of protons from the amide groups was concluded to be a source of the charge carriers in solid polypeptides.
\end{abstract}

KEY WORDS Conductivity / Dielectric Constant / Hydrogen-Deuterium Exchange / Carrier Generation / Poly $(\gamma$-methyl L-glutamate) / Poly $(\gamma$-benzyl L-glutamate) / Poly( $\beta$-benzyl L-aspartate) / Hydrogen Bonds / Glass-Like Transition /

Research on the electric conductivity of nylons and polypeptides has frequently been published. ${ }^{1-17}$ The generally accepted view is that the conduction of these substances is partly ionic and partly electronic ${ }^{5-8}$ and is caused by the transport of charge carriers through continuous chains of hydrogen bonds of the amide groups. Ions supplied from residual impurities have also been considered a source of charge carriers. ${ }^{9,10}$

In previous papers, ${ }^{15-17}$ it was shown that the conductivities of $\operatorname{poly}(\gamma$-methyl L-glutamate) (PMG) and nylon 12 were higher in the direction of the chain of hydrogen bonds than in other directions. We therefore consider that the electric conduction of these polymers depends mainly on the nature of hydrogen bonds and is modified by other factors. In the present paper, attention is directed to the controlling factors for conductivity other than hydrogen bonds. One attempt has also been made to obtain additional experimental evidence that protons from amide hydrogen atoms migrate as charge carriers. PMG, poly$(\gamma$-benzyl L-glutamate) (PBG), and poly( $\beta$-benzyl L-aspartate) (PBA) were used as polymers and their molecular structures are given as follows:

$$
\begin{array}{cccc}
(-\mathrm{NH}-\mathrm{C} H-\mathrm{CO}-)_{p} & \mathrm{PMG} & n=2, & \mathrm{R}=\mathrm{CH}_{3} \\
\left(\mathrm{CH}_{2}\right)_{n} & \text { PBG } & 2, & \mathrm{CH}_{2} \mathrm{C}_{6} \mathrm{H}_{5} \\
\text { OC-OR } & \text { PBA } & 1, & \mathrm{CH}_{2} \mathrm{C}_{6} \mathrm{H}_{5} .
\end{array}
$$

PBG is known to have two solid-state modifications $^{18,19}$ : "Film A" cast from chloroform solution, and "Film B" cast from benzene solution. "Film B" has been argued to have 'stacks' of benzene rings in the side chain and to form a super helical structure. "Film A" of PBG, PMG, and PBA has been reported to have no such side chain structure. The helical structure of PBA is known to be quite unstable. ${ }^{20}$

\section{EXPERIMENTAL}

Ajicote A-2000 was used as the raw PMG material and was supplied by Ajinomoto Co. Ltd., as a 10 weight percent solution. PBG was obtained from Kyowa Hakko Kogyo Co. Ltd., and PBA from Miles Laboratories, Inc. Ajicote A-2000 was diluted by trichloroethylene, whereas PBG and PBA were 


\section{S. Hirota}

Table I. The list of the samples used

\begin{tabular}{|c|c|c|c|c|c|}
\hline \multicolumn{2}{|c|}{ Sample } & Solvent & $\begin{array}{c}\begin{array}{c}\text { Annealing } \\
\text { temperature }\end{array} \\
{ }^{\circ} \mathrm{C}\end{array}$ & $\frac{\text { Thickness }}{\mu \mathrm{m}}$ & \\
\hline \multirow[t]{3}{*}{ PMG } & -1 & $\mathrm{EDC}+\mathrm{PC}+\mathrm{TC}^{\mathrm{a}}$ & 125 & 37 & Ajicote A-2000 \\
\hline & -2 & $\mathrm{EDC}+\mathrm{PC}+\mathrm{TC}^{\mathrm{a}}$ & 120 & 39 & Ajicote A-2000 \\
\hline & -3 & $\mathrm{EDC}+\mathrm{PC}+\mathrm{TC}^{\mathrm{a}}$ & 60 & 30 & Ajicote A-2000 \\
\hline \multirow[t]{3}{*}{ PBG } & -1 & Chloroform & 100 & 15 & \\
\hline & -2 & Benzene & 75 & 9 & Stacked \\
\hline & -3 & Chloroform & 50 & 15 & \\
\hline \multirow[t]{2}{*}{ PBA } & -1 & Chloroform & 100 & 14 & Molecular weight $=3900$ \\
\hline & -2 & Chloroform & 55 & 16 & Molecular weight $=3900$ \\
\hline
\end{tabular}

a EDC, ethylene dichloride; PC, perchloroethylene; TC, trichloroethylene; Ajicote A-2000 was made by dissolving $\mathrm{PMG}$ in EDC and PC.

dissolved in chloroform. Part of the PBG was dissolved in benzene.

Film samples were made from solutions of about 2 to 3 weight percent. The PMG and PBG solutions were cast on quartz plates at room temperature and dried slowly. After being peeled off the quartz plates by immersion in methanol, the films were washed with acetone in an ultrasonic washer. After the films were dried in an ambient atmosphere, a gold electrode provided with a guard ring were vacuumevaporated onto the film. Since the degree of polymerization of the PBA used was low, the film was quite brițtle. Therefore, the PBA films for electrical measurements were made by casting the solution directly onto gold plates, which constituted part of the electrodes. The PBA film used for infrared measurements was made by casting the solution onto $\mathrm{CaF}_{2}$ plates. Its thickness was estimated from the concentration of the solution, the volume poured onto the plates, and the area of the film. The thickness of the film samples was measured with a dial gauge. The samples thus prepared are listed in Table I.

The films were dried and annealed at a pressure less than $10^{-2}$ Torr for a few hours prior to each electrical measurement. The annealing temperatures are shown in Table I. The dc conductivity was measured at various temperatures below the annealing temperature, using a Takeda Riken TR-84M vibrating-reed type electrometer. The conductivity of each sample was determined by subtracting the discharging current from the charging current. The dielectric constant was measured by a General
Radio 1615-A capacitance bridge.

The deuteration of the amide groups in the polypeptides was investigated by infrared spectroscopy, using the method reported by Blout et al. ${ }^{21}$ After thin films of polypeptides were immersed in heavy water $(99.75 \%$ pure, Merck) and maintained at $52^{\circ} \mathrm{C}$, the optical density of the amide II band relative to the amide I band was measured as a function of immersion time.

\section{RESULTS}

\section{Electrical Measurement}

Figure 1 shows Arrhenius plots for the conductivity $\sigma$ of solid PMG, PBG, and PBA. These plots can be represented by the equation

$$
\log \sigma=\text { const }-\Delta H^{*} / R T
$$

where $\Delta H^{*}$ is the activation energy for $\sigma, R$ is the gas constant, and $T$ is the absolute temperature. The values of $\Delta H^{*}$ and the conductivities at $1000 / T=$ 3.0, $\sigma_{1}$, are given in Figure 1. For PMG the temperature dependence of $\log \sigma$ shows a break point at about $0^{\circ} \mathrm{C}$. In the temperature range below the break point, the activation energy is smaller. The conductivity at the break point is of the order of $10^{-17} \mathrm{ohm}^{-1} \mathrm{~cm}^{-1}$. No break point was observed for PBG and PBA possibly since the duration time of the absoption current in the low conductivity region is very large, making it difficult to measure the steady-state conductivity.

It has been reported that the temperature dependence of $\sigma$ of polymers usually exhibits a break 


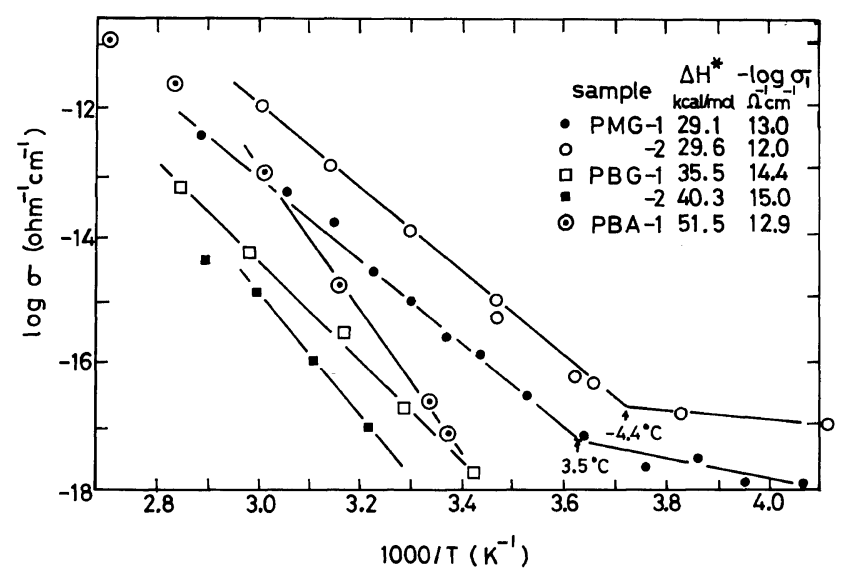

Figure 1. Temperature dependence of polypeptide conductivity. The activation energy in the temperature range near $1000 / T=3.0$ and the conductivity $-\log \sigma_{1}$ are shown in this Figure. $\left(\sigma_{1}\right.$ is the conductivity of polypeptides at $1000 / T=3.0$.)

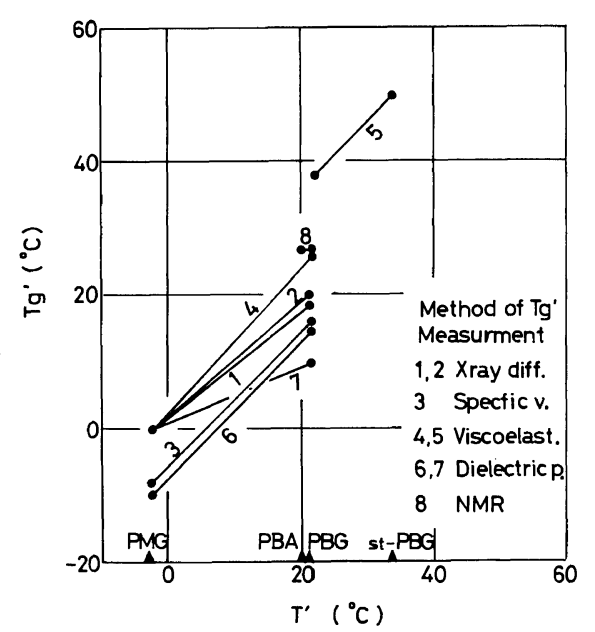

Figure 2. Glass-like transition temperature $T_{\mathrm{g}}{ }^{\prime}$ of polypeptides plotted as a function of $T^{\prime}$. Line numbers and corresponding references are: 1,2 , ref $27 ; 3$, ref 23 ; 4 , ref $26 ; 5$, ref $18 ; 6$, ref $25 ; 7$, ref 28; 8 , ref 24 . Some $T_{\mathrm{g}}{ }^{\prime}$ values were from the figures given in the references.

near the glass transition temperature. ${ }^{22}$ The reported values of $\sigma$ at the break point are scattered around $10^{-17} \mathrm{ohm}^{-1} \mathrm{~cm}^{-1}$. It was found that this value of polyphenylene oxide linearly increased with an increase in the concentration of impurities. ${ }^{22}$ The glass-like transition in polypeptides is attributed to the onset of side-chain motion, and is quite similar to the glass transition of polymers from the standpoint of the temperature dependence of the specific

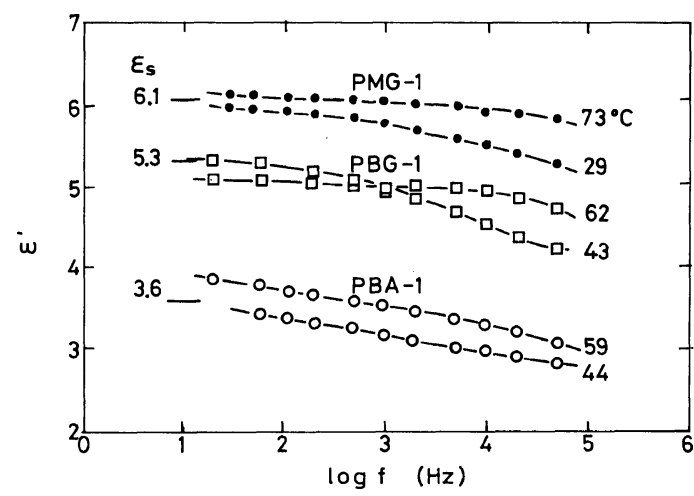

Figure 3. Dielectric constants of polypeptides as a function of frequency at various temperatures. The static dielectric constants are shown on the left side.

volume. ${ }^{23}$ The break point of the conductivity of PMG shown in Figure 1 seems to be related to the glass-like transition. Accordingly, we assumed that the conductivity at the glass-like transition temperature of a pure polypeptide, $\sigma^{\prime}$, was about $10^{-17}$ $\mathrm{ohm}^{-1} \mathrm{~cm}^{-1}$. The difference in $\sigma^{\prime}$ between PMG-1 and PMG-2 probably results from the difference in impurity content. Although the conductivity of polymers at the glass transition temperature should have been $1 \times 10^{-17} \mathrm{ohm}^{-1} \mathrm{~cm}^{-1}$, the values measured at these temperatures of polypeptides were $10^{-17.5} \mathrm{ohm}^{-1} \mathrm{~cm}^{-1}$. The glass-like transition temperatures $T^{\prime}$ of PMG, PBA, PBG, and stacked PGB thus determined by conductivity were estimated to be $-2.7,20.1,21.4$, and $33.3^{\circ} \mathrm{C}$, re- 
spectively. The relation between $T^{\prime}$ and reported glass-like transition temperatures $T_{\mathrm{g}}{ }^{18,23-28}$ is shown in Figure 2, which indicates that $T_{\mathrm{g}}{ }^{\prime}$ and $T^{\prime}$ agree fairly well. Therefore, $T^{\prime}$ and $\sigma^{\prime}$ obtained here are considered to correspond to the glass-like transition.

Figure 3 shows the dielectric constants of polypeptides as a function of frequency at various temperatures. The static dielectric constant $\varepsilon_{\mathrm{s}}$ was estimated from the lowest frequency limit of the observed dielectric constant. The contribution of the conduction loss appearing in the low frequency range $^{29}$ has been omitted. The activation energies for conductivity of the polypeptides are plotted as a function of the reciprocal of $\varepsilon_{\mathrm{s}}$ in Figure 4. The value of $\varepsilon_{\mathrm{s}}$ is temperature dependent, so that the maximum value of $\varepsilon_{\mathrm{s}}$ in this figure was used.

The activation energies of the polypeptides are seen to be inversely proportional to $\varepsilon_{\mathrm{s}}$, although no such clear correlation is found for the other polymers plotted in Figure 4. The intercept of the straight line at $10 / \varepsilon_{\mathrm{s}}=0$ is $8 \mathrm{kcal} \mathrm{mol}^{-1}$. The linear relation for these polypeptides may be due to a property held in common by these polymers such that they possess a chain of hydrogen bonds consisting of amide groups. The data points for nylon 6 and nylon 12, which have the same sort of hydrogen bonds, fall on a line that is the same as that in Figure 4. Along with the fact that the direction of carrier transport in nylon 12 and PMG is parallel to the direction of hydrogen bonds, ${ }^{15,17}$ this finding suggests that these polymers have the same mech- anism of conductivity.

\section{Hydrogen-Deuterium Exchange}

It is often argued that amide hydrogen acts as a charge carrier., ${ }^{1,5,14}$ If the conduction in hydrogen-bonded solid polymers such as polypeptides originates from the transport of protons through the chain of hydrogen bonds, the amide hydrogen should be released from amide groups. Released hydrogen in certain cases may be ionized and thus move as a charge carrier. The release of amide hydrogen was checked by a hydrogen-deuterium exchange experiment.

Figure 5 shows infrared spectra of a thin PMG film before (broken line) and after (solid line) soaking for $460 \mathrm{~h}$ in heavy water at $52^{\circ} \mathrm{C}$. The rate of deuteration was determined as a function of time, and the results are shown in Figure 6. For PMG and PBA, a short initial period of fast exchange rate is followed by a slow deuteration rate. This initial reaction may take place in the peptide groups near either the surface of the film or the ends of the molecules. Since the degree of polymerization of PBA is low, the ratio of the coil region to the helix region must be large. ${ }^{30}$ This fact may account for the large initial decrease of the amide hydrogen content in the PBA film. No deuteration of the amide hydrogen could be seen in PBG, suggesting that the rate of deuteration of the amide groups in the PBG film is much slower than that in the PBA film. A similar trend has been reported for the deuteration of PBG and PBA in solution. ${ }^{31}$ The rate

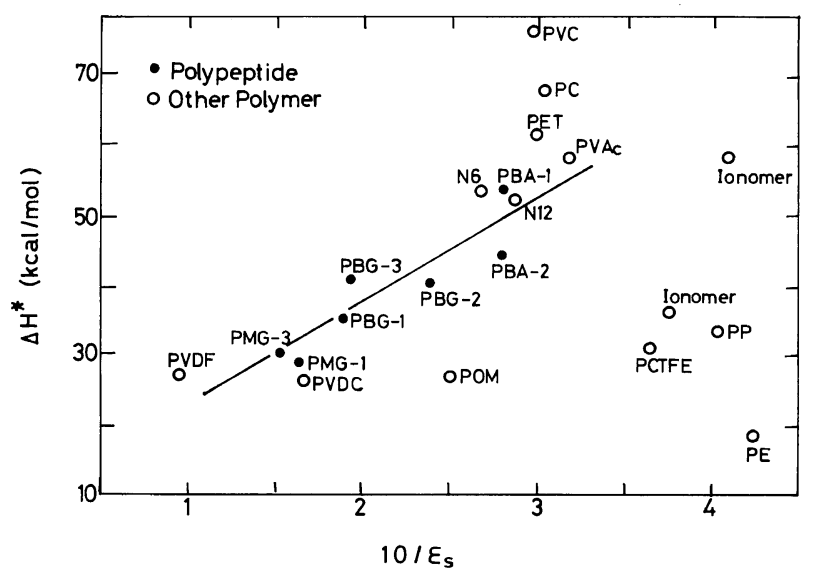

Figure 4. The activation energy of polymers is shown as a function of the reciprocal of the static dielectric constant. The filled circles are for polypeptides. 


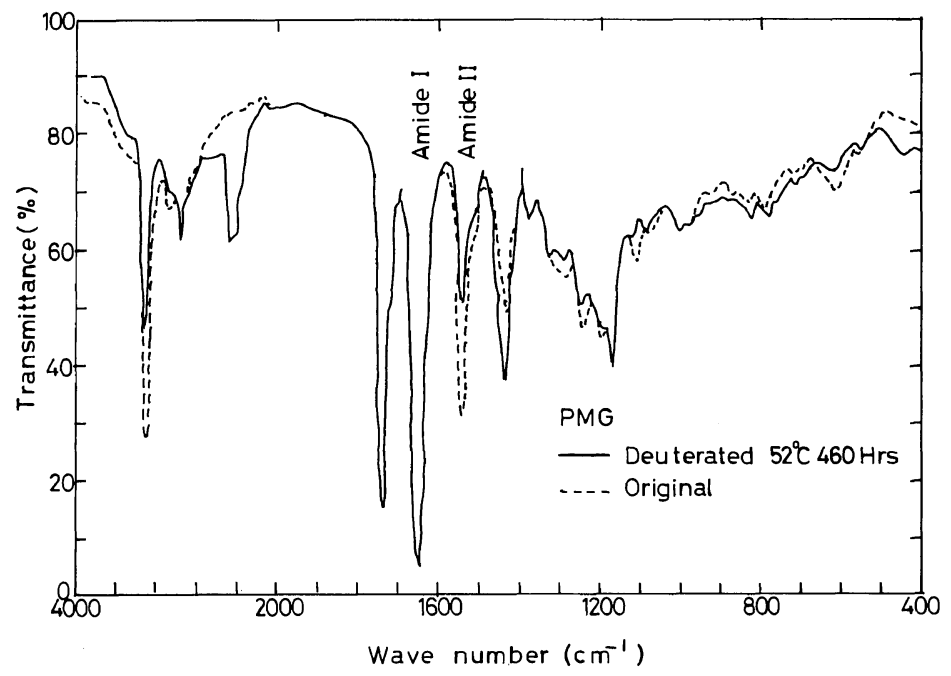

Figure 5. Infrared absorption spectra of deuterated PMG film soaked in heavy water at $52^{\circ} \mathrm{C}$ for $460 \mathrm{~h}$ and that of the original film.

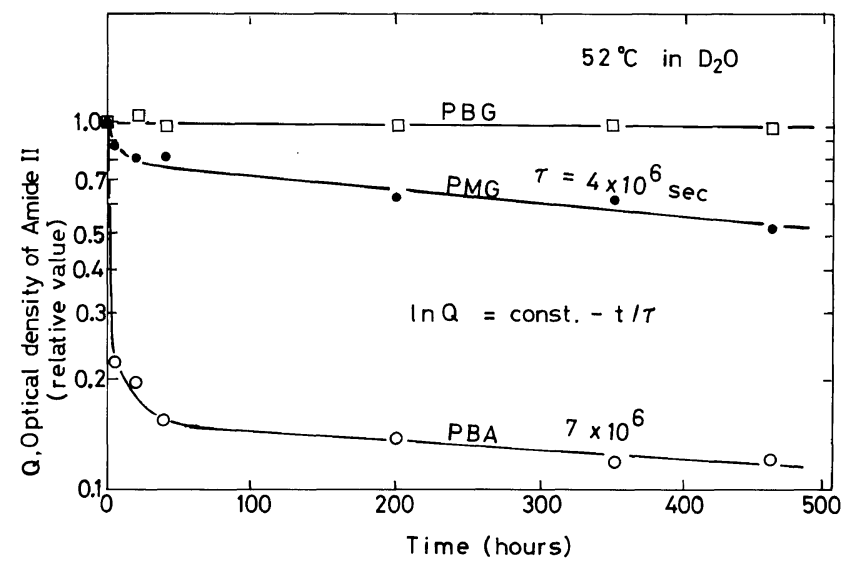

Figure 6. The time dependence of the optical density of amide II absorption. The PMG, PBG, and PBA films were soaked in heavy water maintained at $52^{\circ} \mathrm{C}$.

constants for deuteration in the region of slow exchange rate were $4 \times 10^{6} \mathrm{~s}$ for PMG and $7 \times 10^{6} \mathrm{~s}$ for PBA.

\section{DISCUSSION}

As shown in Figure 4, the activation energy for conductivity varies linearly with the reciprocal of the dielectric constant; i.e.,

$$
\Delta H^{*}=\Delta H_{0}^{*}+A / \varepsilon_{\mathrm{s}}
$$

where $\Delta H_{0}{ }^{*}$ is a constant and the second term represents the contribution of charge separation against the Coulomb force in the continuous medium of the dielectric constant $\varepsilon_{\mathrm{s}}$. Charge carriers may be generated by the self-ionization of amide groups. If the separation distance of the initial ion pair is $r$ and the dielectric constant of the medium surrounding the ion pairs is $\varepsilon_{\mathrm{s}}$, the contribution of the carrier generation to the activation energy can be calculated from eq 2, with $A$ given by

$$
A=q^{2} / 8 \pi \varepsilon_{0} r,
$$

where $q$ is the charge of the carrier and $\varepsilon_{0}$ is the 


\section{S. Hirota}

permitivity of vacuum. If we assume that $q$ is equal to the charge of an electron and equate $\varepsilon_{\mathrm{s}}$ to the observed macroscopic value, we obtain $r$ to be $1.1 \AA$, which indicates that it is not legitimate to use the macroscopic dielectric constant for $\varepsilon_{\mathrm{s}}$ in eq 3 , since the distance $1.1 \AA$ is too small to be considered reasonable. Thus the continuous medium approximation does not hold in this case. More detailed considerations on the electrostatic structure of molecules, such as the distribution and direction of dipoles and charges, must be made.

The constant term $\Delta H_{0}{ }^{*}$ in eq 2 may be regarded as due to the transport of charge carriers. Two mechanisms may be considered for carrier transport. One is associated with the dissociation of hydrogen bonds, followed by the reorientation of the amide groups in polypeptides, i.e., the local helix-coil transformation in the $\alpha$-helix. The dissociation energy for the $\mathrm{CO}-\mathrm{HN}$ hydrogen bond is known to be $8 \mathrm{kcal} \mathrm{mol}^{-1}$. ${ }^{32}$ The reported activation energy of rotation is $8 \mathrm{kcal} \mathrm{mol}^{-1}$ for the CC bond, and $14 \mathrm{kcal} \mathrm{mol}^{-1}$ for the CONH bond in polypeptides. ${ }^{33}$ The activation energy for carrier transport associated with the rotation of the amide group may not be less than the sum of the dissociation and rotation energies, about $20 \mathrm{kcal} \mathrm{mol}^{-1}$ The other mechanism of carrier transport is proton hopping through the chain of hydrogen bonds. The activation energy for this process is not known. We assume that it is equal to $\Delta H_{0}$.

The activation energy for the carrier mobility in PMG measured in a previous paper ${ }^{16}$ was $29 \mathrm{kcal}$ $\mathrm{mol}^{-1}$, which is quite different from the activation energy $\left(8 \mathrm{kcal} \mathrm{mol}^{-1}\right)$ obtained in this paper. This discrepancy can be explained by considering the carrier transport in polypeptides as a multi-step hopping process: a charge carrier is generated initially by the self-ionization of an amide group, the generated carrier then hops many times through the chain of hydrogen bonds, and finally the carrier collides with the opposite charge and disappears. The hopping carrier may behave as a carrier in the free state, while the non-ionized hydrogen of the amide group may be considered as being in the trapped state. The effective mobility of a charge carrier is then given by the mobility of a free carrier multiplied by its fractional hopping time. The activation energy of the apparent mobility may be the sum of the activation energy of mobility and that of carrier generation.
The process of hydrogen-deuterium exchange of amide groups in solid polypeptides is not yet clear. The generally accepted view for this process in solution is that the helix region of polypeptide molecules is in dynamic equilibrium with the coil region. The exchange takes place between the amide hydrogen in the coil region and the deuterium of heavy water. ${ }^{34}$ As shown in the present paper, solid PMG and PBA immersed in heavy water exchange their amide hydrogen with a deuterium of heavy water. In regard to the exchange reaction in the solid state, Praissman and Rupley ${ }^{35}$ have shown that a portion of the hydrogen atoms included in crystalline insulin is exchanged with tritium of tritiated water. The characteristic time constant $\tau$ of hydrogen-deuterium (or tritium) exchange can be determined from the concentration $Q$ of the amide hydrogen using the relation

$$
\log Q=\text { const }-t / \tau \text {. }
$$

The values of $\tau$ in polypeptides and insulin are shown in Table II. The magnitude of $\tau$ in the solid state is larger than that in solution. The values in the solid state are almost constant, being of the order of $10^{7} \mathrm{~s}$. Those in the solution are scattered from $10^{5}$ to $10^{6} \mathrm{~s}$. Thus, the hydrogen-deuterium exchange in the solid state is much slower than that in the solution. The reason for this is that the $\alpha$-helix is stabler in the solid state than in solution and that the concentration of heavy water in the side chain region of polypeptides is lower in the solid state than in solution. The solubility of heavy water in a PMG film at $52^{\circ} \mathrm{C}$ is 1.6 weight percent. The reason for the difference in $\alpha$-helix stability between the solid state and the solution and which of the two factors dominates deuteration is not clear.

Table II. The time constant of hydrogen exchange in various materials in the solid state and in solution

\begin{tabular}{lcc}
\hline & \multicolumn{2}{c}{ Time constant of hydrogen exchange/s } \\
\cline { 2 - 3 } & Solid state & Solution \\
\hline PMG & $4 \times 10^{6}$ & - \\
PBA & $7 \times 10^{6}$ & $2 \times 10^{6 \mathrm{a}}$ \\
Insulin & $9 \times 10^{6 \mathrm{~b}}$ & $5 \times 10^{4 \mathrm{~b}}$ \\
PGA $^{\mathrm{d}}$ & - & $3 \times 10^{5 \mathrm{c}}$ \\
\hline
\end{tabular}

${ }^{\mathrm{a}}$ Reference $32 .{ }^{\mathrm{b}}$ Reference $36 .{ }^{\mathrm{c}}$ Reference $30 .{ }^{\mathrm{d}}$ Poly(Lglutamic acid). 
Another mechanism of hydrogen-deuterium exchange is the diffusion of deuterium atoms directly through the chain of hydrogen bonds, as charge carriers are transported by the hopping process discussed above. Deuterium is taken up by the film as a result of hydrogen-deuterium exchange at the surface of the film. In this case, the diffusion constant of deuterium can be estimated from the thickness of the film (about 3 micrometers) and the time constant of deuteration determined by infrared absorption. The relevant equation is ${ }^{36}$

$Q=\left(8 / \pi^{2}\right) \sum_{n=0}^{\infty}(2 n+1)^{-2} \exp \left[-(2 n+1)^{2}\left(\pi^{2} / d^{2}\right) D t\right]$

where $D$ is the diffusion constant, $d$ is the thickness of the film, and $n$ is a non-negative integer. The diffusion constant of deuterium in the PMG film was estimated to be $9.5 \times 10^{-15} \mathrm{~cm}^{2} \mathrm{~s}^{-1}$. The diffusion constant of water vapour through the side chain region between helices in solid PMG at $25^{\circ} \mathrm{C}$ is reported to be about $10^{-7} \mathrm{~cm}^{-2} \mathrm{~s}^{-1} \cdot{ }^{37}$ Thus, the diffusion of deuterium occurs not through the side chain region but through the chain of hydrogen bonds.

There are still other possibilities for hydrogendeuterium exchange: heavy water diffuses in the side chain region between helices and the hydrogendeuterium exchange takes place at active points of the polypeptide (chain ends and the coil regions). It is clear that the hydrogen atom of an amide group is easily released and transported in the form of a proton.

\section{CONCLUSIONS}

In regard to electric conduction, polypeptides have the following properties in common. The conductivity at the glass-like transition temperature is about $3 \times 10^{-18} \mathrm{ohm}^{-1} \mathrm{~cm}^{-1}$ and its activation energy is linearly dependent on the reciprocal of the static dielectric constant $\varepsilon_{\mathrm{s}}$. The activation energy at the limit of $1 / \varepsilon_{\mathrm{s}}=0$ is $8 \mathrm{kcal} \mathrm{mol}^{-1}$. These properties are considered to arise from the following conduction mechanism: a proton from the amide group becomes a charge carrier and is transported through the chain of hydrogen bonds consisting of amide groups. Hydrogen-deuterium exchange experiments suggest that amide groups easily release hydrogen atoms and that the amide hydrogen is a potential carrier source in polypeptides.

Acknowledgements. The author wishes to thank Dr. S. Saito of Kyushu University and Dr. U. Itoh of Electrotechnical Laboratory for their interest, encouragement, and support. $\mathrm{He}$ also wishes to thank Ajinomoto Co. Ltd., for kindly supplying the Ajicote A-2000.

\section{REFERENCES}

1. W. O. Baker and W. A. Yager, J. Am. Chem. Soc., 64, 2171 (1942).

2. D. W. McCall and W. Anderson, J. Chem. Phys., 32, 237 (1960).

3. D. D. Eley and D. I. Spivey, Trans. Faraday Soc., 57, 2280 (1961).

4. D. A. Seanor, J. Polym. Sci., C, No. 17, 195 (1967).

5. D. A. Seanor, J. Polym. Sci., A-2, 6, 463 (1968).

6. Y. Miyoshi and N. Saito, J. Phys. Soc. Jpn., 24, 1007 (1968).

7. H. Kasica, M. Kryszewski, A. Szymaniski, and M. Wlodarczyk, J. Polym. Sci., A-1, 6, 1615 (1968).

8. M. E. Baird, J. Polym. Sci., A-2, 8, 739 (1970).

9. S. Isoda, H. Miyaji, and K. Asai, Jpn. J. Appl. Phys., 12, 1799 (1973).

10. S. Nakamura, G. Sawa, and M. Ieda, Jpn. J. Appl. Phys., 20, 47 (1981).

11. D. D. Eley and D. I. Spivey, Trans. Faraday Soc., 56, 1432 (1960).

12. K. Hikichi, J. Saito, A. Tsutsumi, and M. Kaneko, Rep. Prog. Polym. Phys. Jpn., 16, 593 (1973).

13. H. Sasabe, H. Oosugi, and S. Saito, Rep. Prog. Polym. Phys. Jpn., 17, 631 (1974).

14. S. Kuga and H. Kambe, Rept. Prog. Polym. Phys. Jpn., 17, 425 (1974).

15. S. Hirota, J. Appl. Phys., 50, 3007 (1979).

16. S. Hirota, Kobunshi Ronbunshu, 36, 653 (1979).

17. S. Hirota, J. Appl. Phys., 53, 3792 (1982).

18. T. Fukuzawa, Y. Uematsu, and I. Uematsu, Polym. J., 6, 431 (1974).

19. T. Watanabe, Y. Tsujita, and I. Uematsu, Polym. J., 7, 181 (1975).

20. H. Kyotani and H. Kanetsuna, J. Polym. Sci., Polym. Phys. Ed., 10, 1931 (1972).

21. E. R. Blout, C. de Lozé, and A. Asadourian, J. Am. Chem. Soc., 83, 1895 (1961).

22. S. Saito, Oyo Buturi, 44, 3 (1975).

23. K. Hikichi, A. Tsutsumi, S. Isozaki, and M. Kaneko, Polym. J., 7, 646 (1975).

24. K. Hikichi, J. Phys. Soc. Jpn., 19, 2169 (1964).

25. A. Tsutsumi, K. Hikichi, T. Takahashi, Y. Yamashita, N. Matsushima, M. Kanke, and M. Kaneko, J. Macromol. Sci.-Phys., B8, 413 (1973).

26. An-Lac Nguyen, Ban The Vu, and G. L. Wilkes, $J$. Macromol. Sci.-Phys., B9, 367 (1974). 
27. N. Matsushima, K. Hikichi, A. Tsutsumi, and M. Kaneko, Polym. J., 7, 44 (1975).

28. N. Matsushima, K. Hikichi, A. Tsutsumi, and M. Kaneko, Polym. J., 8, 88 (1976).

29. S. Saito, S. Hirota, and S. Sasabe, Rep. Prog. Polym. Phys. Jpn., 17, 399 (1974).

30. M. Nakanishi, M. Tsuboi, A. Ikegami, and M. Kanehisa, J. Mol. Biol., 64, 363 (1972).

31. E. M. Bradbury, A. R. Downie, A. Elliott, and W. E. Hanby, Proc. R. Soc., London, Ser. A, 259, 110 (1960).

32. D. S. Trifan and J. F. Terenzi, J. Polym. Sci., 28, 443 (1958).
33. T. Miyazawa, "Infrared Spectra and Helical Conformations," in "Poly- $\alpha$-amino Acids," G. D. Fasman, Ed., Marcel Dekker Inc., New York, 1967, p 82.

34. S. W. Englander, "Hydrogen Exchange," in "Poly- $\alpha$ amino Acids," G. D. Fasman, Ed., Marcel Dekker, Inc., New York, 1967, p 339.

35. M. Praissman and J. A. Rupley, J. Am. Chem. Soc., 86, 3584 (1964)

36. A. S. Carpenter and D. F. Twiss, Rubber Chem. Technol., 13, 326 (1940).

37. A. Takizawa, T. Hamada, H. Okada, and H. Nonoyama, J. Appl. Polym. Sci., 18, 1443 (1974). 